\section{Toolkit to support dental practices reopening}

Dentally has created a useful guide covering some key elements that dental practices will need to consider as they begin to open their practice and see patients (https://bit. ly/3c082SD). This includes an essential toolkit of measures to support dentists and their staff during this time, by saving time, encouraging social distancing and managing the administration needed to reopen the practice to deliver the care patients need.

The toolkit will be available to all of Dentally customers and includes the following:

Outbound Telephone Calling and Tasks Lists. Telephone calls can now be made within Dentally, and this new functionality has now been fully integrated to allow you to make calls direct from the patient record.

The outbound telephone calling feature within Dentally complements another new feature: Tasks List. This time-saving function will ensure tasks such as calls, correspondence, admin and patient follow-up is completed and not forgotten during the busy day-to-day running of a dental practice - whether your staff are working on site or remotely.

Contactless Reception. Another exciting new feature from Dentally is self check-in SMS for patients arriving at the practice. Patients can be sent an SMS just prior to their appointment time at the practice. The patient can click on this link and check themselves in as they arrive on site. They can also complete or update their medical history - so you can run a contactless reception to support social distancing.

To then close the loop in the process, following the patient's appointment, Dentally has added payment link functionality. This means that the practice can now send an SMS with a secure link that allows the patient to pay their bill by clicking on the link, and making a payment using a credit/ debit card, Apple Pay, etc.

Chat. Dentally understands that keeping in touch with all staff is essential to retaining clear communication and continuity between members of teams who are split between working in the practice on site and those working remotely. The chat feature allows you to send secure and instant messages within the software.

Templates and Reports. In order to help practitioners compile and put all the patient correspondence needed to advise patients of the new procedures, remote check in, payment links and appointment guidance, the Dentally team have created a number of templates that can be used. In addition, the reports within the system are also helpful in preparation of a return to seeing patients in person. Guidance on these and some saved filters are available to all customers in a dedicated collection of COVID-19 support documents for the software.

If you would like more information on how Dentally can make your life easier as you begin to think about returning to practice, then contact hello@dentally.co to talk to the team. Visit https://dentally.co/.

\section{A complete disinfection range for dentistry}

EndoSan produce highly effective, broad spectrum hydrogen peroxide-based disinfectants that form no harmful by-products or residuals - degrading into water and oxygen, whilst offering unrivalled stability. It is chlorine and alcohol free, with no corrosive effects. EndoSan is a safe biocide that is truly biodegradable. It can be used for constant or shock dosing in dental unit water lines, fogging, surface disinfection, manual dosing, and hand sanitisation.

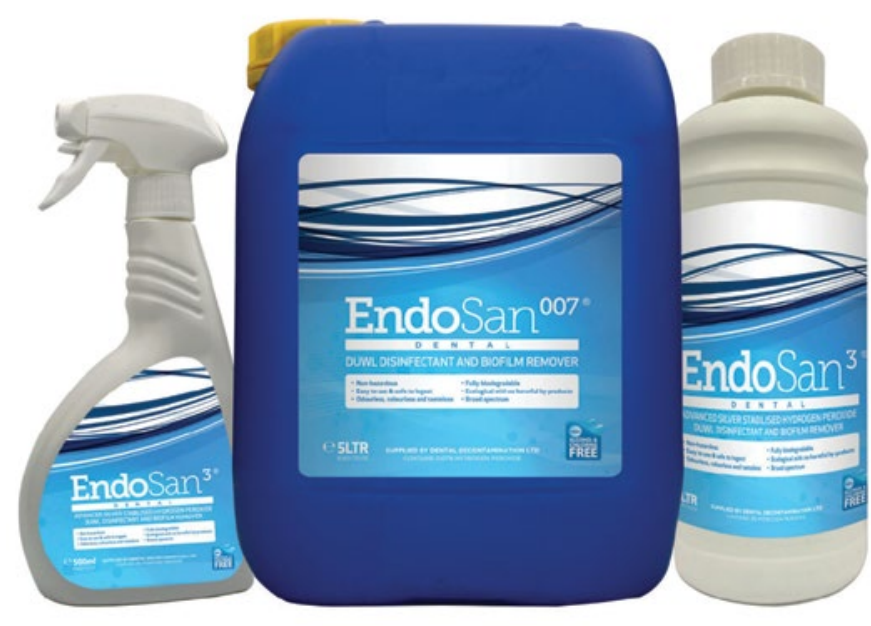

Endosan 3 is a surface disinfectant that is completely odourless, tasteless, and colourless making it the perfect application to wipe down cabinets, chairs, and all other hard surfaces within the dental practice. Endosan 3 comes in a $500 \mathrm{ml}$ bottle with two spray settings which is safe and easy for the user to apply.

Endosan 007 is a dental unit water line disinfectant that can be dispensed straight into the chairside bottle with no need to mix any potentially harmful chemicals. With just three uses of the hand pump (dosing $40 \mathrm{ml}$ ) the 1 litre chair side bottle will be ready for use by topping up with either tap water or purified water. Endosan 007 can also be used to sanitise RO systems by clearing out all biofilm that may have built up, which will help prolong the life of future filters.

For more Endosan products such as Hand Sanitisers or to purchase the Endosan range call 01253 736355, email account@ dentaldecontamination.co.uk or visit shop.dentaldecontamination.co.uk.

\section{Free educational toolkit for dentists}

When the pandemic first arrived Dominic Hassall, one of the top clinical dental lecturers and educators in the world, put together a free Dominic Hassall Training Institute (DHTI) Educational Toolkit for dentists. The uptake has been fantastic, and dentists have been amazed at how much clinical information could be released free.

The DHTI Educational Toolkit has almost four hours of online video lectures, plus articles and free resources to help all dentists to be stronger as we navigate through this crisis.

To obtain Dominic's free educational toolkit visit https:// dominic-hassall-training.co.uk/toolkit/. 